(The 3rd ICBS-2013)

\title{
PRODUCTION OF ANTIBODY IgY ANTI-C-Myc IN CHICKEN EGGS
}

\author{
Salomo Hutahaean \\ Department of Biology, Faculty of Mathematics and Natural Sciences, Universitas Sumatera Utara, \\ JIn. Bioteknologi. No 1, Kampus USU, Medan 20155. \\ Email: salomo@usu.ac.id
}

\begin{abstract}
Bioinformatics analysis has been performed on c-Myc peptide sequences to predict its epitopes in order to design antigen for IgY anti-c-Myc production in chickens. Peptide sequences for human c-Myc protein obtained from the NCBI database were analyzed with ProPred-1 softwares. Manual screening has been conducted on the prediction results. One candidate of c-Myc epitope, EQKLISEEDL, have been selected and prepared as an antigen, by adding one molecule of Cystein to the peptide, and conjugated it with Keyhole limpet hemocyanin (KLH). Twelve hens, aged 22-24 weeks, were immunized a week after their first eggs were produced. Antigen diluted in aquabidest and emulsified with Freund's Complete Adjuvant. The suspension was injected into the area of the chicken breast, subcutaneously or intramuscularly, with a dose of $0.1 \mathrm{mg}$ for the first immunization. Booster injection performed on days 10, 20, and 30 with the same volume of emulsion, but using Incomplete Freund's Adjuvant and the amount of antigen half of the first immunization. Extraction of IgY from egg has been carried out using kits from Gallus Immunotech. IgY levels quantified using a spectrophotometer, absorbance measured at $280 \mathrm{~nm}$. The results showed that chicken antibody production gave rise to a sigmoid curve pattern, just like in mammals. The IgY content of the egg was stable at high levels (>5 mg / $\mathrm{ml}$ yolk) after the third immunization. In the intramuscular group, the immune response seems to work earlier than in the subcutaneous group, but eventually the subcutaneous group reaches the same level, and even higher. In general, from an egg produced after the second booster it can be extracted at least $50 \mathrm{mg}$ of IgY. Higher yields could be obtained when IgY was extracted from egg 2 to 3 days after the third booster.
\end{abstract}

Key words: epitope prediction, c-Myc, IgY antibody, ProPred-1

\section{INTRODUCTION}

Antibody was initially used for therapy, but now it has been used in many fields of research for various purposes. Antibody can be used in the characterization and quantification of specific protein, or in verification of protein localization. Its usefulness as tools for many biomedical researchers is due to its ability to recognize and bind to a specific target molecule. Many antibodies today have been used in diagnostic applications, such as in pregnancy test kits, in protein microarrays, in immunohistochemistry, and ELISA. Increase widespread use has led to increased demand for antibodies (Anonymous, 2011). Unfortunately, for developing countries like Indonesia most of domestic demand for antibodies are met by imports. Attempts to produce antibodies domestically face problem of expertise, equipment and high production costs.

Most of antibodies in the market today are produced in the mammalian bodies. There were several disadvantages of this mode of production. Firstly, this mode of production resulting in relatively small quantity of antibody. It is only a very small quantity of antibody that can be extracted from blood. Secondly, there is a problem with cross-reaction phenomenon, when the antibodies from one mammalian species is used as a diagnostic tool for other mammals. Thirdly, there is ethical implication when produce antibodies in mammalian

ISSN 2413-0877 @ 2015 The Authors.

Published by KnowledgeE Publishing Services This is an open access article under the CC BY-NC-ND license (http://creativecommons.org/licenses/by-nc-nd/4.0)

Selection and Peer-review under responsibility of the 3rd ICBS-2013

Doi http://dx.doi.org/10.18502/kls.v2i1.168 
bodies. The technique usually generates stress and pain in the animals, due to injection of antigen and adjuvant and repeated blood sampling in large volume for antibodies extraction. These problems should be solved by production of monoclonal antibodies in vitro. However, the production of monoclonal antibodies requires special expertise and very expensive laboratory equipment.

As an alternative to the production of antibodies in the mammalian, Polson (1990) has developed techniques to produce antibody in poultry. With this new technique, the results obtained are larger. In the chicken, $20-30 \mathrm{mg}$ of antigen was able to produce $130 \mathrm{mg}$ of specific antibodies. IgY antibodies provide the added advantage of minimal cross-reaction when used as tools for detection of mammalian protein (Gassmann et al., 1990). In addition, since $\mathrm{lg} Y$ is extracted from eggs, the production system is essentially generated no pain and low stress-effects in experimental animals.

Production of antibodies, both in mammal and in poultry, requires precise design of an immunogen that able to trigger body immune response. As is generally known, for generating an immune response it does not need to use the whole protein as an antigen, but quite a small segment of the protein known as antigenic determinant or epitope. Determination of epitope of a protein is a very complex job. Factors to be considered in epitope prediction and design of the immunogen include: i). Epitope length (generally in the range of 10-20 residues), ii). Hydrophobicity, surface orientation, and flexibility, iii). Targeting the $\mathrm{N}$-terminus or C-terminus, iv). Continuous and discontinuous epitopes, v). Algorithms to predict protein characteristics such as hydrophilicity / hydrophobicity and secondary structure of the region including the alpha-helix, beta sheet, vi). The selection process of the sequence, such as avoiding common sequence motifs (e.g. RGD motif, helix-loop-helix sequence, GTP binding sites, and SH2 domains) that can cause cross-reactivity, and vii). Coupling of peptides to carrier protein (Ponomarenkom and van Regenmortel., 2009). Because of the importance of immunogen design, epitopes prediction has become a specialized field of immunology which is rapidly evolving. Currently, prediction of epitope can be done efficiently with the help of computer programs. There are many computer programs available with the ability to predict epitopes. Some of them rely solely on the information which can be obtained from the linear sequence of protein antigen, for example ABCpred (Saha \& Raghava, 2006) and COBEpro (Sweredoski \& Baldi, 2009), while others depend on the availability of threedimensional structures, such as the CEP program (Kulkarni-Kale et al., 2005) and DiscoTope (Andersen \& Lund, 2006). There are also programs that can analyze both 3-D protein structure and peptide linear sequence, such as program Epitopia (Rubinstein et al., 2009), and programs targeting the MHC-binding peptides such as ProPred (Singh \& Raghava, 2001). In this paper, production of IgY anti-c-Myc in chickens were reported. The c-Myc antibody was chosen to produce, because it is widely used in many research areas, among others, in relation to health, developmental biology, and research on R\&D and evaluation of plantbased drugs for cancer therapy (He et al., 2008). The c-Myc protein is generally activated in many tumor cells and plays an important role in proliferation, differentiation, and cell cycle. 


\section{MATERIALS AND METHODS \\ ProPred-1 epitope prediction}

Sequence for analysis were mRNA of the human c-Myc protein in Fasta format. The sequences of 454 amino acid peptide size were obtained from the NCBI database (RefSeq/ protein_id = NP_002458.2):

MDFFRVVENQQPPATMPLNVSFTNRNYDLDYD SVQPYFYCDEEENFYQQQQQSELQPPAPSEDIWKKFELLPTPPLS PSRRSGLCSPSYVAVTPFSLRGDNDGGGGSFS TADQLEMVTELLGDMVNQSFICDPDDETFIKNIIIQDCMWSGFSAA AKLVSEKLASYQAARKDSGSPNP ARGHSSTSSLYLQDLSAAASECIDPSVVFPYPLNDSSSPKSCASQDSSAFSPSSDS LLSSTESSPQGSPELVLHEETPPTTSSDSEEEQEDEEEIDVV SVEKRQAPGKRSESGSPSAGGHSKPPHSPLVLKRCTH QHNYAAPPSTRKDYPAAKRVKLDSVRVLRQ ISNNRKCTSPRSSDTEENVKRRTHNVLEQRRNELKRSFFALRDQIPE LENNEKAPKVVILKKATAYILSVQAEEQKLISEEDLLRKRREQLKHKLEQLRNSCA

A personal computer connected to the internet was used. Analysis was accomplished online, by connecting to http://www.imtech.res.in/raghava/propred1/ (ProPred-1 server). ProPred-1 predict epitopes that can bind to alleles of MHC class I molecules. This program uses a matrix-based antigen sequence for $47 \mathrm{MHC}$ class-I alleles. ProPred-1 provides the option for the analysis of one allele, the combination of multiple alleles, or analysis of all 47 alleles.

Data of human c-Myc peptide sequence was copy-pasted to the ProPred-1 server in Fasta format. Twelve MHC class I alleles were selected to be analyzed. A threshold score of $4 \%$ (default) and output display in HTML-II format were selected. The program processed the data for several minutes (time depends on the length of the sequence entered). Prediction results were screened manually to obtain a candidate of epitope with 10 amino acids size. Five upper ranked of sequences resulting from software prediction were manually screened to obtain one candidate of epitope with 10 amino acids size. Candidate wherever possible have to meet the following criteria: i). Does not contain Proline, Cystein, and Methionine, ii). Possessed more exposed (e) than buried (b) amino acids, iii.) Not from helical regions of the protein. iv). Does not contain $\mathrm{N}$-terminal Aspergine. v). Does not contain aspartic acid in combination with Glycine, Proline, and Serine. vi). No multiple Serine and Proline, and vii). No serial order of Glutamine, Isoleusine, Leusine, Phenylalanine, Threonine, Tyrosine, or Valin (Angeletti, 1999; Anonymous, 2012).

\section{Immunization protocol}

Twelve hens, 22-24 weeks of age, purchased from a breeder and kept in battery cages. Animals were fed commercial feed (chicken par-G-pellets) and received tap water ad libitum. The immunization was started on the day 7 after chickens started laying eggs. Synthetic c-Myc peptide (Genescript) $(0.1 \mathrm{mg})$ were diluted in aquabidest, until the final volume of $750 \mathrm{ml}$. Antigen solution was then emulsified with an equal volume of Freund's Complete Adjuvant (Pacific Immunology., A\#5003). For subcutaneous route of immunization, suspension was injected into the space under the skin in the breast area. Subsequent immunization was applied on days 10,20, and 30 with the same volume of emulsion, but using Incomplete Freund's Adjuvant and the amount of antigen half of the first. For the intramuscular immunization route, the suspension was injected into the breast muscle. Eggs were collected daily, marked and stored at $4^{\circ} \mathrm{C}$ until use. Eggs that collected prior to immunization were used as controls. 


\section{$\lg \mathrm{Y}$ extraction and quantification}

IgY antibody was extracted using the EggsPress IgY Purification Kit (Gallus Immunotech Inc.). Yolk was separated from the white egg using an egg separator. Yolk then rinsed with distilled water and 5 volumes of Reagent $\mathrm{A}\left(4^{\circ} \mathrm{C}\right)$ were added very slowly. Suspension stirred until it well blended and then kept in refrigerator at $4^{\circ} \mathrm{C}$ overnight. Suspension then centrifuged at $4000 \mathrm{Xg}$ for $20 \mathrm{~min}$ at $4^{\circ} \mathrm{C}$. Supernatant was collected into a beaker glass and an equal volume of reagent $B\left(4^{\circ} \mathrm{C}\right)$ was added while stirring gently for 2 minutes. The suspension centrifuged again, at $4000 \mathrm{Xg}$ for $20 \mathrm{~min}$ at $4^{\circ} \mathrm{C}$. After that, the supernatant was discarded and the precipitate obtained ( $\mathrm{IgY}$ ) was diluted in PBS. Absorbance was measured with a spectrophotometer at $280 \mathrm{~nm}$ for quantification. IgY antibody was filter sterilized and stored in the refrigerator.

\section{RESULT AND DISCUSSION Epitope prediction}

Analysis of c-Myc protein sequences with the program ProPred-1 produces several epitope candidates. After a manual screening procedure, one segment of 10 amino acids (EQKLISEEDL) was selected and developed into an antigen. The epitope is located in number 426-434 of c-Myc peptide. It has a high immunogenicity scale at 7 of its amino acid, moderate for one amino acid, and low for other amino acids. Amino acids with low immunogenicity scale in this segment is Leusine, which is not an exposed amino acid. A synthetic peptide was purchased with custom by sending information to GenScript company, USA. The information provided as a basis for the synthesis were the peptide segments (EQKLISEEDL), the addition of Cystein to the terminal of the sequence, and conjugation to carrier proteins. Because the peptides are generally too small to generate sufficient immune response to produce antibodies, the peptide was conjugated to a carrier protein to stimulate T-helper cells, which induces $B$ cells to generate immune responses. Keyhole limpet hemocyanin (KLH), a copper-core molecules containing non-heme proteins found in arthropods and mollusks, were used as carrier protein. KLH conjugate was chosen because its higher imunogenicity compared to other carrier proteins.

\section{$\lg Y$ production}

Synthetic antigen containing a short sequence of peptide resulted from the computer program prediction (ProPred-1) was capable of eliciting an immune response in chickens (Figure 1). The antibody production gives a sigmoid curve pattern, just like in mammals (Hanly et al., 1995).

In figure $1, D_{0}$ eggs (collected before immunization) was evidently also contains antibodies at low rates $(1,084 \mathrm{mg} / \mathrm{ml})$. This means that, naturally chicken body received various antigens and responded to them by producing antibodies. However, the amount of antibody produced by natural antigen is relatively small compared to the amount produced by the immunization process (Harskin, 2001).

In the intramuscular group, the immune response seems to work earlier than in the subcutaneous group, but eventually the subcutaneous group reaches the same level, and even higher. Two days after the first immunization $\left(D_{2}\right), \lg Y$ level has reached $4.646 \mathrm{mg} / \mathrm{ml}$ yolk, 


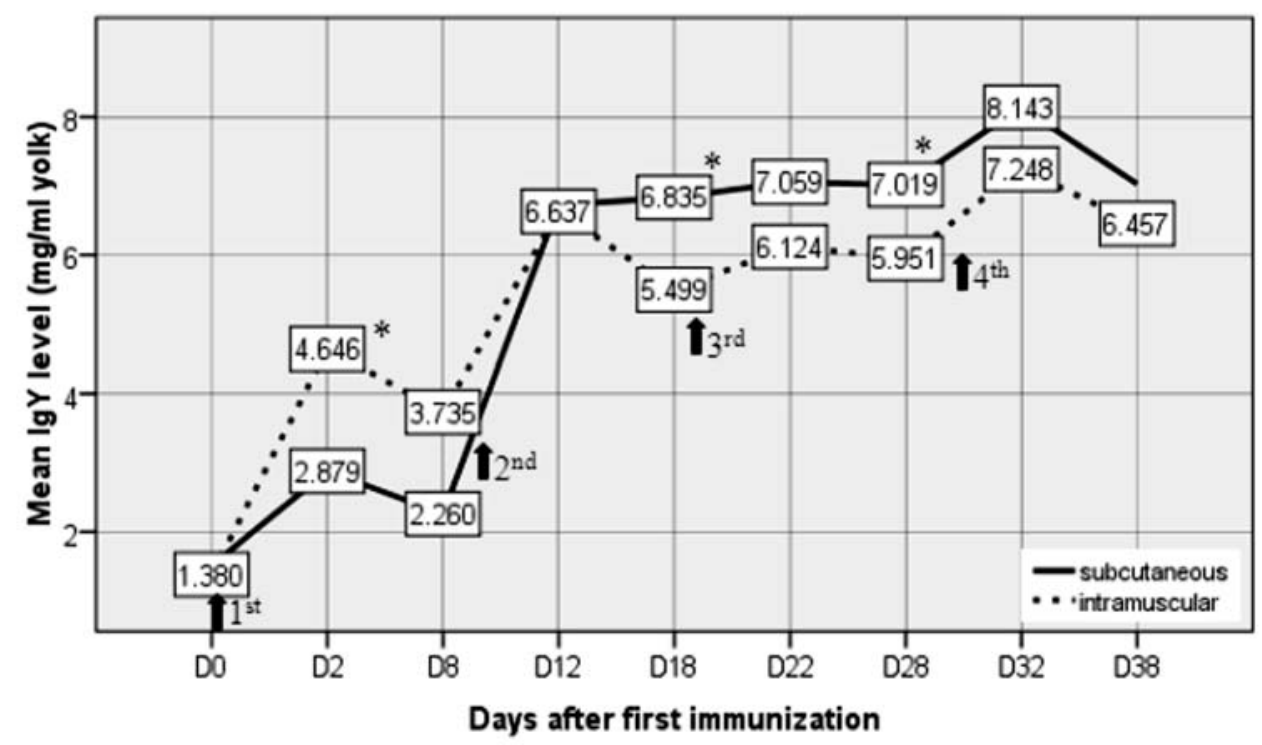

Figure 1. The expression of $\mathrm{lg} Y$ concentration $(\mathrm{mg} / \mathrm{ml}$ yolk) in eggs monitored for 38 days after immunization of chickens by synthetic antigen designed from human cMyc peptide sequence. (Arrows: immunization schedules; Asterics: significant difference $(P<0.05)$ by $t$-test for equality of means between subcutaneous and intramuscular group for the same D).

while in subcutaneous group it was only $2.876 \mathrm{mg} / \mathrm{ml}(\mathrm{P}<0.05)$. After that time IgY levels decreased in both groups and only increased after the second booster was given $\left(D_{10}\right)$ and reached the same level in $D_{12}(P>0.05)$. After third immunization $\left(D_{20}\right)$, IgY level was constantly higher in the subcutaneous group where it was relatively stable at a level of 6 to $7 \mathrm{mg} /$ $\mathrm{ml}$ yolk as detected in $\mathrm{D}_{18}$ and $\mathrm{D}_{28}$ eggs $(\mathrm{P}<0.05)$. Based on these differences, and the fact that subcutaneous immunization causes minimal pain to the chicken, it is suggested that in the process of $\lg Y$ production, it is better if the immunization was given by subcutaneous rather than intramuscular injection. On subcutaneous route, chicken experiment showed no pain and rejection, in contrast to intramuscular injections that always causes chicken floundered and struggled.

In general, from an egg produced after the second booster it can be extracted at least $50 \mathrm{mg}$ of $\mathrm{lg} Y$. Higher yields could be obtained when lgY was extracted from egg 2 to 3 days after the third booster.

\section{ACKNOWLEDGEMENT}

Thanks to the Directorate General of Higher Education of Indonesia, which has funded this research through a scheme of Fundamental Research, Year 2012-2013 budget.

\section{REFERENCES}

Andersen, H.P., M. Nielsen, and O. Lund. 2006. Prediction of residues in discontinuous Bcell epitopes using protein 3D structures. Protein Sci 15 (11): 2558-2567.

Angeletti, R.H. 1999. Design of Useful Peptide Antigens. J Biomol Tech. No.10: 2-10. Anonymous. 2011. Pharmaceuticals \& Biotech Industry Global Report - 2011. IMAPS. Gassmann, M., P. Thommes, T. Weiser, and U. Hubscher. 1990. Efficient production of chicken egg yolk antibodies against a conserved mammalian protein. The FASEB Journa/ Vol. 


\section{4: 2528-2532.}

Hanly, W.C., J.E. Artwohl, and B.T. Bennett. 1995. Review of Polyclonal Antibody Production Procedures in Mammals and Poultry. ILAR Journa/V37(3) 1995.

Harkins, K.R. 2001. Antibody Purification Methods. In: Howard, G.C., and D.R. Bethell (editors). Basic Methods In Antibody Production And Characterization. CRC Press LLC, USA. 141-168.

He, C., H. Hu, R. Braren, S-Y. Fong, A. Trumpp, T.R. Carlson, and R. A. Wang. 2008. c-myc in the hematopoietic lineage is crucial for its angiogenic function in the mouse embryo. Development 135: 2467 - 2477.

Kulkarni-Kale, U., S. Bhosle, A.S. Kolaskar. 2005. CEP: a conformational epitope prediction server. Nucleic Acids Res (33 Web Server):W168-171.

Polson, A. 1990. Isolation of IgY from the yolks of eggs by a chloroform polyethylene glycol procedure. Immunol Invest 19: 253-258.

Ponomarenko, J.V., and M.H.V. van Regenmortel. 2009. B-Cell Epitope Prediction. In: Gu, J., and P.E. Bourne. Structural Bioinformatics Second Edition. John Wiley \& Sons, Inc. 849-79.

Rubinstein, N.D., I. Mayrose, E. Martz, and T. Pupko. 2009. Epitopia: a web-server for predicting B-cell epitopes. BMC Bioinformatics, 10:287.

Saha, S., and G.P. Raghava. 2006. Prediction of continuous B-cell epitopes in an antigen using recurrent neural network. Proteins 65(1): 40-48.

Sweredoski, M.J., and P. Baldi. 2009. COBEpro: a novel system for predicting continuous B-cell epitopes. Protein Engineering Design \& Selection, 22(3):113-120.

Singh, H., and G.P.S. Raghava. 2001. ProPred: Prediction of HLA-DR binding site. Bioinformatics Application Notes. Vol. 17 No. 12: 1236-1237. 